\title{
Valutazione economica dello studio TNT
}

Lorenzo G. Mantovani $^{(1)}$, Sabato Montella ${ }^{(1)}$, Anna Citarella ${ }^{(1)}$, Simona de Portu ${ }^{(1)}$

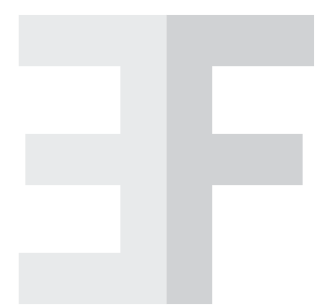

\begin{abstract}
Introduction: the Treating to New Targets (TNT) study showed that intensive lipid-lowering therapy with atorvastatin $80 \mathrm{mg} /$ die provides significant clinical benefit beyond that afforded by atorvastatin $10 \mathrm{mg} / \mathrm{die}$ in patients with stable coronary heart disease (CHD).

Objective: our aim was to investigate the economic consequence of high dose of atorvastatin in Italian patients with stable coronary heart disease (CHD).

Methods: data were derived from the Intensive Lipid Lowering with Atorvastatin in Patients with Stable Coronary Disease (TNT) study. We conducted a cost-effectiveness analysis, comparing high dose of atorvastatin (80 $\mathrm{mg} / \mathrm{die})$ versus usual-dose of atorvastatin $(10 \mathrm{mg} / \mathrm{die})$ in the perspective of the Italian National Health Service. We identified and quantified medical costs: drug costs according to the Italian National Therapeutic Formulary and hospitalizations were quantified based on the Italian National Health Service tariffs (2006). Effects were measured in terms of patients free from any event. We considered an observation period of 4.9 years.

The costs borne after the first 12 months were discounted using an annual rate of $3 \%$. We conducted one and multi-way sensitivity analyses on unit cost and effectiveness.

Results: the cost of atorvastatin $10 \mathrm{mg}$ or $80 \mathrm{mg}$ therapy over the 4.9 years period amounted to approximately $€ 1.6$ millions and $€ 2.5$ millions per 1,000 patients respectively. The total cost of atorvastatin high dose was about $€ 3.7$ millions, the incremental cost per patient free from event is about $€ 12,600$.

Discussion: this evaluation found that atorvastatin therapy is cost-effective. Sensitivity analysis shows that cost consequences parameters are substantially sensitive to fluctuation.
\end{abstract}

Keywords: atorvastatin, coronay heart disease, cost, Italian National Health System

\section{INTRODUZIONE}

Lo studio "Intensive lipid lowering with atorvastatin in patients with stable coronary disease" (TNT) recentemente ha dimostrato come la terapia con atorvastatina a dosaggi elevati, atta a ridurre il colesterolo LDL a livelli inferiori a $100 \mathrm{mg} / \mathrm{dl}$ è associata a sostanziali benefici clinici in pazienti con coronaropatia [1].

Il numero di eventi è inferiore nel gruppo di pazienti trattati con atorvastatina $80 \mathrm{mg}$ rispetto al gruppo trattato con atorvastatina 10 mg. Se confrontata con la terapia a dosi standard, la terapia con alte dosi di atorvastatina riduce il rischio di eventi cerebrovascolari del $22 \%$. Non si sono invece rilevate differenze statisticamente significative nei due gruppi per quanto riguarda il numero di ictus emorragici come primo evento.

Scopo del presente studio è valutare, sulla base di tali considerazioni, l'impatto economico in Italia dell'utilizzo di dosi elevate versus dosi standard di atorvastatina in pazienti con malattia coronarica.

\section{METODI}

Le informazioni cliniche sono state tratte dallo studio "Intensive lipid lowering with atorvastatin in patients with stable coronary disease" (TNT) [1] che ha valutato gli effetti dell'atorvastatina nella prevenzione secondaria di eventi cardiovascolari maggiori in pazienti con evidenze di coronaropatie.

Nello studio TNT 10.001 pazienti di età compresa tra i 35 e i 75 anni, con una storia pregressa di infarto miocardico acuto, angina e rivascolarizzazione coronariche sono stati randomizzati a ricevere atorvastatina $10 \mathrm{mg} / \mathrm{die}$ (5.006) o atorvastatina $80 \mathrm{mg} / \mathrm{die}$ (4.995).

L'end-point primario dello studio è stato il tempo all'insorgenza di uno dei seguenti eventi: morte coronarica, ospedalizzazione per infarto miocardico acuto, arresto cardiaco non fatale e ictus fatale e non [1].

La valutazione economica è stata effettuata tramite un' analisi di costo/efficacia [2-3] che ha confrontato le conseguenze economiche e cliniche dell'utilizzo di dosi standard $(10 \mathrm{mg} / \mathrm{die})$ versus dosi elevate $(80 \mathrm{mg} / \mathrm{die})$ di atorvastatina. L'analisi è stata eseguita secondo la prospettiva
${ }^{(1)} \mathrm{CIRFF}$, Centro Interdipartimentale di Ricerca in

Farmacoeconomia e Farmacoutilizzazione, Università degli Studi di Napoli Federico II 


\section{Tabella I}

Costi unitari

* Ipotesi di un flat price di atorvastatina $80 \mathrm{mg}$ rispetto ad atorvastatina $40 \mathrm{mg}$

\begin{tabular}{lcc}
\hline \multicolumn{1}{c}{ Variabili } & Costi unitari $(\boldsymbol{\epsilon})$ & Referenza \\
\hline Eventi cardiovascolari & $4.567,46$ & {$[8]$} \\
Atorvastatina $10 \mathrm{mg}$ & 0,99 & {$[6]$} \\
Atorvastatina $80 \mathrm{mg}$ & 1,56 & {$[6]^{\star}$} \\
\hline
\end{tabular}

del Servizio Sanitario Nazionale (SSN), adottando il profilo temporale dello studio TNT, che indica 4,9 anni di follow-up mediano.

Tutte le analisi sono riferite ad ipotetiche coorti di 1.000 pazienti [4-5].

L'analisi di costo/efficacia è stata condotta calcolando il rapporto incrementale di costo/efficacia (ICER) come rapporto tra la differenza nei costi delle due alternative in relazione alla differenza di efficacia. L'ICER è espresso come costo per paziente libero da evento cardiovascolare.

\section{Effetti}

Sono stati identificati, misurati e quantificati gli effetti mediante l'utilizzo delle evidenze dello studio TNT, espresse come eventi cardiovascolari evitati e paziente libero da evento cardiovascolare.

\section{Costi}

Coerentemente con la prospettiva d'analisi, sono stati identificati, misurati e quantificati i costi diretti sanitari.

Per il calcolo del costo della terapia farmacologica si è utilizzato lo schema terapeutico seguito nello studio TNT. Tale schema prevedeva che i pazienti che rispettavano i criteri di eleggibilità venivano randomizzati a ricevere 10 $\mathrm{mg} /$ die di atorvastatina o $80 \mathrm{mg} /$ die di atorvastatina. Il 7,2\% dei pazienti in trattamento con $80 \mathrm{mg}$ di atorvastatina e il 5,3\% dei pazienti in trattamento con $10 \mathrm{mg}$ di atorvastatina ha interrotto la terapia a causa di effetti collaterali.

Il costo della terapia farmacologica è stato quantificato sulle basi della dose giornaliera e della durata del trattamento utilizzando il prezzo di cessione all'SSN dei farmaci, ipotizzando un flat price di atorvastatina $80 \mathrm{mg}$ rispetto ad atorvastatina $40 \mathrm{mg}$ [6].

Le tariffe ospedaliere associate ai DRG (Diagnosis Related Group) [7] sono state utilizzate come base per il calcolo dei costi delle ospedalizzazioni, secondo una scelta consistente con la prospettiva adottata. In Tabella I si riporta la stima del costo per evento cardiovascolare quantificata da Bustacchini e colleghi [8], i quali, potendo esistere più tariffe in relazione alla gravità dello stato di salute del paziente alla dimissione, hanno stimato il costo medio pesato per evento, ponderando le tariffe per la frequenza degli eventi in accordo con le più recenti informazioni disponibili sui ricoveri ospedalieri in Italia [9].

I costi associati agli eventi avversi da farmaco non sono stati considerati nell'analisi in quanto non erano disponibili informazioni dettagliate a riguardo.

I costi oltre i primi 12 mesi sono stati scontati con un tasso del $3 \%$; per semplicità solo i risultati scontati sono riportati. I costi sono espressi in euro 2006.

\section{Sensibilità}

Per determinare la solidità dell'analisi è stata anche effettuata un'analisi di sensibilità su quelli che costituiscono i principali parametri: il prezzo dei farmaci $( \pm 10 \%)$ e i costi delle ospedalizzazioni e delle procedure $( \pm 10 \%)$.

Un'ulteriore analisi di sensibilità è stata condotta sulle procedure di sconto facendo variare il tasso di sconto annuale dal 5\% allo $0 \%$ [10-12].

\section{RISULTATI}

Lo studio TNT intendeva dimostrare come la somministrazione di atorvastatina ad elevati dosaggi al fine di ridurre i livelli di LDL al di sotto dei $100 \mathrm{mg} / \mathrm{dl}$ sia associata a benefici clinici sostanziali in pazienti con malattia coronarica.

L'utilizzo di atorvastatina $80 \mathrm{mg} /$ die porta ad una riduzione relativa pari al $22 \%$ per quanto riguarda gli end-point primari quali: morte per malattia coronarica, infarto miocardico, arresto cardiaco, ictus fatale e non (HR 0,78; 95\% CI, 0,69-0,89; $\mathrm{P}<0,001)$.

Il costo della terapia con atorvastatina 10 $\mathrm{mg} /$ die e $80 \mathrm{mg} /$ die nei 4,9 anni è stato rispettivamente di circa 1,6 milioni di euro e di 2,5 milioni di euro per 1.000 pazienti. Il costo totale delle ospedalizzazioni per eventi cardiovascolari è stato stimato pari a 1,4 milioni di euro nel gruppo trattato con atorvastatina $10 \mathrm{mg}$ e pari a 1,2 milioni di euro nel gruppo trattato con atorvastatina $80 \mathrm{mg}$ (Tabella II).

Il costo totale dovuto all'utilizzo di terapia con atorvastatina ad alti dosaggi è di circa 3,7 milioni di euro per 1.000 pazienti trattati per un periodo di 4,9 anni, con un rapporto incrementale di costo/efficacia di 12.594 euro per paziente libero da evento (Tabella II).

Si è osservata una variazione del rapporto incrementale al variare del costo del farmaco e delle ospedalizzazioni (Tabella III). L'ICER 


\begin{tabular}{lccccc}
\hline \multirow{2}{*}{ Variabili } & \multicolumn{2}{c|}{ Atorvastatina $\mathbf{1 0 ~} \mathbf{~ m}$ * } & \multicolumn{2}{c}{ Atorvastatina $\mathbf{8 0}$ mg* } & Differenza* \\
\cline { 2 - 5 } & Numero & Costi totali & Numero & Costi totali & \\
\hline Costi totali per ospedalizzazioni & 335 & $1.443 .526,09$ & 281 & $1.210 .838,30$ & $-232.687,79$ \\
Costo terapia farmacologica & & $1.627 .891,30$ & & $2.540 .655,30$ & $912.764,00$ \\
Costi totali & & $3.071 .417,39$ & & $3.751 .493,60$ & $680.076,21$ \\
Numero di eventi cardiovascolari & 335 & & 281 & & 54 \\
Costo per paziente libero da evento & & & & & $12.594,00$ \\
\hline
\end{tabular}

Tabella II

Analisi dei costi (in euro), valori scontati al 3\%

* Dati riferiti ad una ipotetica coorte di 1.000 soggetti

varia da 14.284,31 a 10.903,7 euro per paziente libero da evento al variare del costo giornaliero di atorvastatina; quando si applica una variazione al costo delle ospedalizzazioni, l'ICER varia tra $12.163,10$ a $13.024,91$ euro.

\section{DISCUSSIONE}

Non essendo disponibili studi simili di confronto a livello italiano, la presente analisi costituisce la prima valutazione economica dello studio TNT adattato alla realtà italiana.

Si è dimostrato come il trattamento con elevate dosi di atorvastatina porta ad una riduzione dei costi diretti a carico dell'SSN, anche se non tale da compensare il costo addizionale dovuto al trattamento con atorvastatina $80 \mathrm{mg}$.

Lo studio presenta alcuni potenziali limiti: sono calcolati, infatti, solo i costi delle ospedalizzazioni e non i costi associati alla riabilitazione e alla gestione del paziente fuori dalla struttura ospedaliera. Tali informazioni, attualmente non accessibili, potrebbero modificare sostanzialmente il rapporto di costo/efficacia: un'analisi economica condotta direttamente sui pazienti dello studio TNT e riportata al $56^{\circ}$ Congresso Annuale dell'American College of Cardiology da Mark e colleghi [13] indica come, a fronte di dati comparabili con quelli italiani per la terapia farmacologica, il differenziale nei costi totali tra i due gruppi sia minimo e il rapporto di costo/efficacia stimato direttamente sui partecipanti dello studio clinico sia più favorevole di quello stimato nel presente lavoro.

Una seconda limitazione è rappresentata dal fatto che le conseguenze indirette di un evento cardiovascolare, quali la perdita o il guadagno di produttività, o conseguenze intangibili, quali la compromissione della qualità di vita associata allo stato di salute, non sono state incluse. Il motivo è legato al fatto che queste informazioni non erano a nostra disposizione. L'analisi del possibile nesso tra la terapia con statine e la variazione di produttività dei pazienti costituisce un tema di ricerca molto interessante.

Comunque è possibile ipotizzare che, in considerazione delle ospedalizzazioni più fre-

\begin{tabular}{ccc}
\hline Descrizione & Risultati $(\boldsymbol{\epsilon})$ & Variazione $\%$ \\
\hline Prezzo atorvastatina $\pm 10 \%$ & $14.284,31-10.903,7$ & $\pm 13,42$ \\
Costo ospedalizzazioni $\pm 10 \%$ & $12.163,10-13.024,91$ & $\pm 3,42$ \\
\hline
\end{tabular}

Tabella III

Analisi di sensibilità

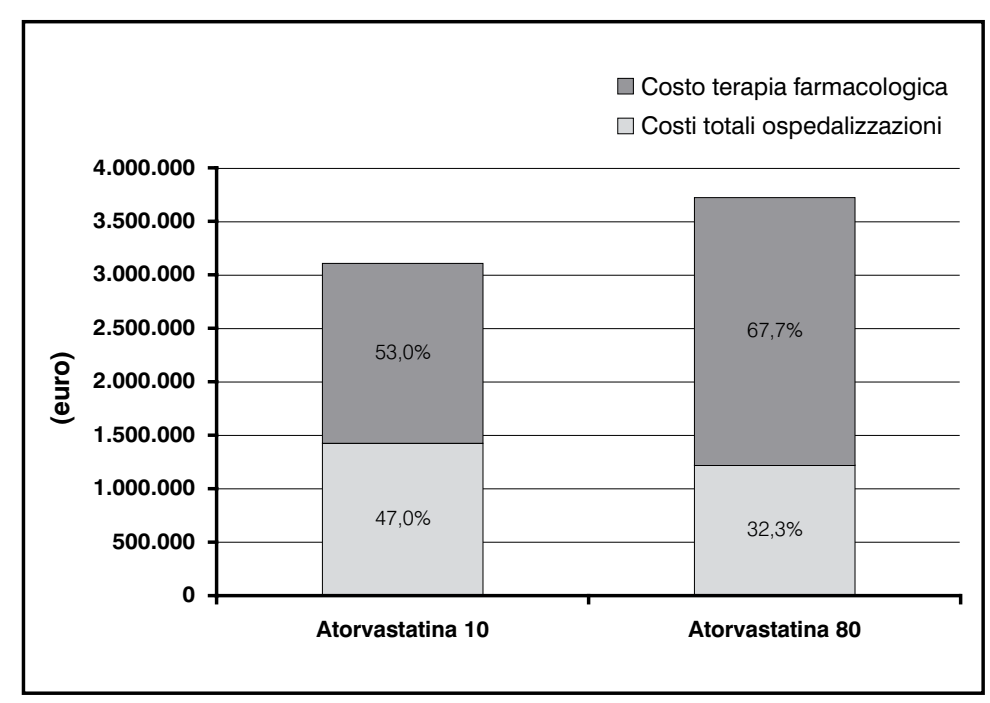

Figura 1

Costo per 1.000 soggetti per 4,9 anni

quenti, $\mathrm{i}$ pazienti in terapia con atorvastatina $10 \mathrm{mg}$ abbiano subito una maggiore riduzione nella capacità lavorativa: secondo tale ipotesi, l'introduzione nella valutazione economica dei costi indiretti aumenterebbe i benefici economici derivanti dall'utilizzo di atorvastatina a dosi elevate.

È anche interessante notare che il costo della terapia farmacologica è stato probabilmente sovrastimato. Infatti, per mancanza di informazioni relative alla durata del ricovero, non è stato possibile tenere in considerazione il fatto che il costo della terapia farmacologica per il periodo della durata del ricovero è compreso nelle tariffe DRG, per cui una parte dei costi relativi alla terapia sono stati quantificati due volte. 


\section{BIBLIOGRAFIA}

1. LaRosa JC, Grundy SM, Waters DD, Shear C, Barter P, et al. Intensive lipid lowering with atorvastatin in patients with stable coronary disease. $N$ Engl J Med 352; 1425-35. Epub 2005 Mar 8

2. Weinstein Mc, Stason WB. On the foundations of cost-effectiveness analysis for health and medical practices. N Engl J Med 1977; 296: 716-2

3. Drummond MF, Stoddart GL, Torrance GW. Methods for economic evaluation of Health Care Programmes. Oxford: Oxford University Press, 1997

4. Mantovani LG, Belisari A, Dobrilla G. Valutazione economica del lansoprazolo nel trattamento dei pazienti affetti da malattia da reflusso esofageo. Pharmacoeconomics Italian Research Articles 1999; 1: 43-51

5. Scalone L, Mantovani LG. Valutazione economica della terapia con lisinopril ad alto verso basso dosaggio nel trattamento dei soggetti con scompenso cardiaco cronico. Pharmacoeconomics Italian Research Articles 2002; 4; 45-55.

6. Informatore Farmaceutico. Milano: OEMF, 2006

7. Decreto ministeriale 14/12/94 in tema di "Tariffe delle prestazioni di assistenza ospedaliera". Supplemento ordinario alla Gazzetta Ufficiale n. 209, 8/09/1997

8. Bustacchini S, Ruffo P, Mantovani LG. Economic evaluation of primary prevention of CVD events with statins in Italy. Value Health 2004; 7: 696

9. http://www.ministerosalute.it/programmazione/sdo/sdo.jsp

10. Briggs A, Sculpher M, Buxton M. Uncertainty in the economic evaluation of health care technologies: the role of sensitivity analysis. Health Econ 1994; 3: 95-104

11. Garattini L, Grilli R, Scopelliti D, Mantovani L. A proposal for Italian guidelines in pharmacoeconomics. Pharmacoeconomics 1995; 7:1-6

12. Capri S, Ceci A, Terranova L, Merlo F, Mantovani LG. Guidelines for economic evaluation in Italy: recommendation from the Italian group of pharmacoeconomic studies. Drug Inf J 2001; 35: 189-201

13. Mark DB, Knight JD, Cowper PA, Davidson-Ray L, Anstrom KJ. High-dose Atorvastatin-better value for money: the treating to new targets trial United States economic substudy in 5.308 stable coronary heart disease patients. J Am Coll Cardiol 2007; 50 (Suppl 1): 290A 